\title{
Numerical Methods for Solving a Hereditary Equation of Hyperbolic Type
}

\author{
V. G. Pimenov ${ }^{1}$ and E. E. Tashirova ${ }^{1}$
}

Received September 22, 2011

\begin{abstract}
A family of grid methods is constructed for the numerical solution of a wave equation with delay of general form; the methods are based on the idea of separating the current state and the history function. A theorem on the order of convergence of the methods is obtained by means of embedding into a general difference scheme with aftereffect. Results of calculating test examples with constant and variable delays are presented.

Keywords: numerical methods, wave equation, delay, difference schemes, interpolation, extrapolation, order of convergence.
\end{abstract}

DOI: $10.1134 /$ S008154381305012X

\section{INTRODUCTION: PROBLEM STATEMENT}

Evolution equations, including equations of hyperbolic type, are used in many mathematical models of the real world; they may contain delay effects of various kinds. Presently, questions of the qualitative theory for such equations are actively studied [1]. However, numerical algorithms for hereditary evolution equations are insufficiently developed. Certain difference schemes for equations of parabolic type with delay of general form were studied in [4]. The approach of this paper is based on the application of the general theory of difference schemes [5] and the theory of numerical methods for functional differential equations $[2,3]$. In this paper, the ideas of [4] are extended to equations of hyperbolic type with one space variable.

Consider the wave equation with heredity effect

$$
\frac{\partial^{2} u}{\partial t^{2}}=a^{2} \frac{\partial^{2} u}{\partial x^{2}}+f\left(x, t, u(x, t), u_{t}(x, \cdot)\right): \quad t_{0} \leq t \leq T, \quad 0 \leq x \leq X
$$

with boundary conditions

$$
u(0, t)=g_{1}(t), \quad u(X, t)=g_{2}(t): \quad t_{0} \leq t \leq T
$$

and initial conditions

$$
u(x, t)=\varphi(x, t): \quad 0 \leq x \leq X, \quad t_{0}-\tau \leq t<t_{0} .
$$

Here, $u(x, t)$ is the required function; $\left.u_{t}(x, \cdot)=\{u(x, t+\xi),-\tau \leq \xi \leq 0)\right\}$ is the history function of the required function to the moment $t ; \tau$ is the value of delay; $f\left(x, t, u(x, t), u_{t}(x, \cdot)\right)$ is

\footnotetext{
${ }^{1}$ Institute of Mathematics and Computer Science, Ural Federal University, pr. Lenina 51, Yekaterinburg, 620000 Russia

emails: Vladimir.Pimenov@usu.ru, Linetisa@yandex.ru
} 
a functional defined on $[0, X] \times\left[t_{0}, T\right] \times \mathbb{R} \times Q$, where $Q=Q[-\tau, 0)$ is the set of the functions $u(\xi)$ that are piecewise continuous on $[-\tau, 0)$ with a finite number of discontinuity points of the first kind and right continuous at the discontinuity points; and $\|u(\cdot)\|_{Q}=\sup _{\xi \in[-\tau, 0)}|u(\xi)|$.

We assume that the functional $f$ and functions $g_{1}, g_{2}, \varphi$ are such that the problem has a unique solution $u(x, t)[1]$.

\section{DIFFERENCE METHOD}

Let us divide the interval $[0, X]$ into parts with step $h=X / N$, where $N$ is an integer. Consider the points $x_{i}=i h, i=0,1 \ldots, N$. Divide the interval $\left[t_{0}, T\right]$ into parts with step $\Delta$. Assume that $m=\tau / \Delta$ is an integer.

Consider the points $t_{j}=t_{0}+j \Delta, j=-m, \ldots, M$. We will denote approximations of the exact solution $u\left(x_{i}, t_{j}\right)$ by $u_{i}^{j}$.

Introduce the discrete history to the moment $t_{j}$ for each fixed $i:\left\{u_{i}^{k}\right\}^{j}=\left\{u_{i}^{k}: j-m \leq k \leq j\right\}$.

A mapping $I:\left\{u_{i}^{k}\right\}^{j} \rightarrow v_{i, j}(\cdot) \in Q[-\tau, \Delta]$ will be called an interpolation-extrapolation operator for the discrete history.

We say that an interpolation-extrapolation operator has error order $p$ on the exact solution if there exist constants $C_{1}$ and $C_{2}$ such that

$$
\left|v_{i, j}(t)-u\left(x_{i}, t\right)\right| \leq C_{1} \max _{j-m \leq k \leq j}\left|u_{i}^{k}-u\left(x_{i}, t_{k}\right)\right|+C_{2} \Delta^{p}
$$

for all $i=0, \ldots, N, j=0, \ldots, M$, and $t \in\left[t_{j}-\tau, t_{j+1}\right]$.

For $0 \leq s \leq 1$, consider a family of methods with the weights

$$
\begin{gathered}
\frac{u_{i}^{j+1}-2 u_{i}^{j}+u_{i}^{j-1}}{\Delta^{2}}=s a^{2} \frac{u_{i-1}^{j+1}-2 u_{i}^{j+1}+u_{i+1}^{j+1}}{h^{2}}+s a^{2} \frac{u_{i-1}^{j-1}-2 u_{i}^{j-1}+u_{i+1}^{j-1}}{h^{2}} \\
+(1-2 s) a^{2} \frac{u_{i-1}^{j}-2 u_{i}^{j}+u_{i+1}^{j}}{h^{2}}+F_{i}^{j}\left(v_{i, j}(\cdot)\right), \quad i=1, \ldots N-1, \quad j=0, \ldots M-1,
\end{gathered}
$$

with boundary conditions $u_{0}^{j}=g_{1}\left(t_{j}\right), u_{N}^{j}=g_{2}\left(t_{j}\right)$ and initial conditions $u_{i}^{j}=\varphi\left(x_{i}, t_{j}\right)$, where $-m \leq j \leq 0$. Here, $F_{i}^{j}\left(v_{i, j}(\cdot)\right)$ is a functional defined on $Q[-\tau, T]$, Lipschitz continuous in the variable $v_{i, j}(\cdot)$ with constant $L_{F}$, and connected with the functional $f\left(x_{i}, t_{j}, u_{i}^{j}, v_{i, j}(\cdot)\right)$.

For $s=0$, we obtain an explicit scheme. For other $s, 0<s \leq 1$, for each fixed $j$, the system is linear tridiagonal with respect to $u_{i}^{j+1}$ with diagonal dominance; hence, it can be effectively solved by the tridiagonal matrix algorithm.

The residual (without interpolation) of the method is the value

$$
\begin{gathered}
\psi_{i}^{j}=\frac{u\left(x_{i}, t_{j+1}\right)-2 u\left(x_{i}, t_{j}\right)+u\left(x_{i}, t_{j-1}\right)}{\Delta^{2}}-s a^{2} \frac{u\left(x_{i-1}, t_{j+1}\right)-2 u\left(x_{i}, t_{j+1}\right)+u\left(x_{i+1}, t_{j+1}\right)}{h^{2}} \\
-s a^{2} \frac{u\left(x_{i-1}, t_{j-1}\right)-2 u\left(x_{i}, t_{j-1}\right)+u\left(x_{i+1}, t_{j-1}\right)}{h^{2}} \\
-(1-2 s) a^{2} \frac{u\left(x_{i-1}, t_{j}\right)-2 u\left(x_{i}, t_{j}\right)+u\left(x_{i+1}, t_{j}\right)}{h^{2}}-F_{i}^{j}\left(u_{t_{j}}\left(x_{i}, \cdot\right)\right) .
\end{gathered}
$$

We will say that the residual has order $h^{p_{1}}+\Delta^{p_{2}}$ if there exists a constant $C$ such that $\left|\psi_{i}^{j}\right| \leq C\left(h^{p_{1}}+\Delta^{p_{2}}\right)$ for all $i=1, \ldots N-1, j=0, \ldots M-1$. 
Theorem 1. Suppose that the exact solution of problem (1.1)-(1.3) has continuous partial derivatives up to the fourth order and $F_{i}^{j}\left(v_{i, j}(\cdot)\right)=f\left(t_{j}, x_{i}, u_{i}^{j}, v_{i, j}(\cdot)\right)$. Then, for every $0 \leq s \leq 1$, the residual has order $h^{2}+\Delta^{2}$.

Proof. The residual is defined by the equality

$$
\begin{gathered}
\psi_{i}^{j}=\frac{u\left(x_{i}, t_{j+1}\right)-2 u\left(x_{i}, t_{j}\right)+u\left(x_{i}, t_{j-1}\right)}{\Delta^{2}}-s a^{2} \frac{u\left(x_{i-1}, t_{j+1}\right)-2 u\left(x_{i}, t_{j+1}\right)+u\left(x_{i+1}, t_{j+1}\right)}{h^{2}} \\
-s a^{2} \frac{u\left(x_{i-1}, t_{j-1}\right)-2 u\left(x_{i}, t_{j-1}\right)+u\left(x_{i+1}, t_{j-1}\right)}{h^{2}}-(1-2 s) a^{2} \frac{u\left(x_{i-1}, t_{j}\right)-2 u\left(x_{i}, t_{j}\right)+u\left(x_{i+1}, t_{j}\right)}{h^{2}} \\
-f\left(t_{j}, x_{i}, u\left(x_{i}, t_{j}\right), u_{t_{j}}\left(x_{i}, \cdot\right)\right) .
\end{gathered}
$$

Expand the function $u(x, t)$ in a Taylor series in a neighborhood of the points $\left(x_{i}, t_{j}\right),\left(x_{i}, t_{j+1}\right)$, and $\left(x_{i}, t_{j-1}\right)$. We obtain the following equalities for the values of the function at these points:

$$
\begin{gathered}
u\left(x_{i}, t_{j+1}\right)=u\left(x_{i}, t_{j}\right)+\frac{\partial u}{\partial t}\left(x_{i}, t_{j}\right) \Delta+\frac{1}{2} \frac{\partial^{2} u}{\partial t^{2}}\left(x_{i}, t_{j}\right) \Delta^{2}+\frac{1}{6} \frac{\partial^{3} u}{\partial t^{3}}\left(x_{i}, t_{j}\right) \Delta^{3}+O\left(\Delta^{4}\right), \\
u\left(x_{i}, t_{j-1}\right)=u\left(x_{i}, t_{j}\right)-\frac{\partial u}{\partial t}\left(x_{i}, t_{j}\right) \Delta+\frac{1}{2} \frac{\partial^{2} u}{\partial t^{2}}\left(x_{i}, t_{j}\right) \Delta^{2}-\frac{1}{6} \frac{\partial^{3} u}{\partial t^{3}}\left(x_{i}, t_{j}\right) \Delta^{3}+O\left(\Delta^{4}\right), \\
u\left(x_{i-1}, t_{j}\right)=u\left(x_{i}, t_{j}\right)-\frac{\partial u}{\partial x}\left(x_{i}, t_{j}\right) h+\frac{1}{2} \frac{\partial^{2} u}{\partial x^{2}}\left(x_{i}, t_{j}\right) h^{2}-\frac{1}{6} \frac{\partial^{3} u}{\partial x^{3}}\left(x_{i}, t_{j}\right) h^{3}+O\left(h^{4}\right), \\
u\left(x_{i+1}, t_{j}\right)=u\left(x_{i}, t_{j}\right)+\frac{\partial u}{\partial x}\left(x_{i}, t_{j}\right) h+\frac{1}{2} \frac{\partial^{2} u}{\partial x^{2}}\left(x_{i}, t_{j}\right) h^{2}+\frac{1}{6} \frac{\partial^{3} u}{\partial x^{3}}\left(x_{i}, t_{j}\right) h^{3}+O\left(h^{4}\right), \\
u\left(x_{i-1}, t_{j+1}\right)=u\left(x_{i}, t_{j+1}\right)-\frac{\partial u}{\partial x}\left(x_{i}, t_{j+1}\right) h+\frac{1}{2} \frac{\partial^{2} u}{\partial x^{2}}\left(x_{i}, t_{j+1}\right) h^{2}-\frac{1}{6} \frac{\partial^{3} u}{\partial x^{3}}\left(x_{i}, t_{j+1}\right) h^{3}+O\left(h^{4}\right), \\
u\left(x_{i+1}, t_{j+1}\right)=u\left(x_{i}, t_{j+1}\right)+\frac{\partial u}{\partial x}\left(x_{i}, t_{j+1}\right) h+\frac{1}{2} \frac{\partial^{2} u}{\partial x^{2}}\left(x_{i}, t_{j+1}\right) h^{2}+\frac{1}{6} \frac{\partial^{3} u}{\partial x^{3}}\left(x_{i}, t_{j+1}\right) h^{3}+O\left(h^{4}\right), \\
u\left(x_{i-1}, t_{j-1}\right)=u\left(x_{i}, t_{j-1}\right)-\frac{\partial u}{\partial x}\left(x_{i}, t_{j-1}\right) h+\frac{1}{2} \frac{\partial^{2} u}{\partial x^{2}}\left(x_{i}, t_{j-1}\right) h^{2}-\frac{1}{6} \frac{\partial^{3} u}{\partial x^{3}}\left(x_{i}, t_{j-1}\right) h^{3}+O\left(h^{4}\right), \\
u\left(x_{i+1}, t_{j-1}\right)=u\left(x_{i}, t_{j-1}\right)+\frac{\partial u}{\partial x}\left(x_{i}, t_{j-1}\right) h+\frac{1}{2} \frac{\partial^{2} u}{\partial x^{2}}\left(x_{i}, t_{j-1}\right) h^{2}+\frac{1}{6} \frac{\partial^{3} u}{\partial x^{3}}\left(x_{i}, t_{j-1}\right) h^{3}+O\left(h^{4}\right) .
\end{gathered}
$$

Substituting these relations into the formula for $\psi_{i}^{j}$, we obtain

$$
\begin{gathered}
\psi_{i}^{j}=\frac{\partial^{2} u}{\partial t^{2}}\left(x_{i}, t_{j}\right)+O\left(\Delta^{2}\right)-s a^{2}\left(\frac{\partial^{2} u}{\partial x^{2}}\left(x_{i}, t_{j+1}\right)+O\left(h^{2}\right)\right)-s a^{2}\left(\frac{\partial^{2} u}{\partial x^{2}}\left(x_{i}, t_{j-1}\right)+O\left(h^{2}\right)\right) \\
-(1-2 s) a^{2}\left(\frac{\partial^{2} u}{\partial x^{2}}\left(x_{i}, t_{j}\right)+O\left(h^{2}\right)\right)-f\left(t_{j}, x_{i}, u\left(x_{i}, t_{j}\right), u_{t_{j}}\left(x_{i}, \cdot\right)\right) .
\end{gathered}
$$

Expand the function $\frac{\partial^{2} u}{\partial x^{2}}(x, t)$ in a Taylor series in a neighborhood of the point $\left(x_{i}, t_{j}\right)$. This yields the following relations:

$$
\begin{aligned}
& \frac{\partial^{2} u}{\partial x^{2}}\left(x_{i}, t_{j+1}\right)=\frac{\partial^{2} u}{\partial x^{2}}\left(x_{i}, t_{j}\right)+\frac{\partial^{3} u}{\partial t \partial x^{2}}\left(x_{i}, t_{j}\right) \Delta+O\left(\Delta^{2}\right), \\
& \frac{\partial^{2} u}{\partial x^{2}}\left(x_{i}, t_{j-1}\right)=\frac{\partial^{2} u}{\partial x^{2}}\left(x_{i}, t_{j}\right)-\frac{\partial^{3} u}{\partial t \partial x^{2}}\left(x_{i}, t_{j}\right) \Delta+O\left(\Delta^{2}\right) .
\end{aligned}
$$


Then,

$$
\psi_{i}^{j}=\frac{\partial^{2} u}{\partial t^{2}}\left(x_{i}, t_{j}\right)-a^{2}\left(\frac{\partial^{2} u}{\partial x^{2}}\left(x_{i}, t_{j}\right)\right)-f\left(t_{j}, x_{i}, u\left(x_{i}, t_{j}\right), u_{t_{j}}\left(x_{i}, \cdot\right)\right)+O\left(\Delta^{2}+h^{2}\right) .
$$

By equation (1.1), $\psi_{i}^{j}=O\left(\Delta^{2}+h^{2}\right)$.

The theorem is proved.

Denote the error of the method at the nodes by $\varepsilon_{i}^{j}=u\left(x_{i}, t_{j}\right)-u_{i}^{j}$. We say that the method converges if $\varepsilon_{i}^{j} \rightarrow 0$ as $h \rightarrow 0, \Delta \rightarrow 0$ for all $i=0, \ldots, N, j=0, \ldots M$. We say that the method converges with order $h^{p}+\Delta^{q}$ if there exists a constant $C$ independent of $\Delta$ and $h$ such that the inequality $\left|\varepsilon_{i}^{j}\right| \leq C\left(h^{p}+\Delta^{q}\right)$ holds for all $i=0, \ldots, N, j=0, \ldots M$.

To study the convergence, we apply the technique of abstract difference schemes with aftereffect.

\section{INVESTIGATION OF THE CONVERGENCE}

Let us investigate the convergence of method (2.1) by means of embedding it into the general difference scheme [2-4]. We will consider problems with the homogeneous boundary conditions

$$
u(0, t)=u(X, t)=0, \quad t_{0} \leq t \leq T .
$$

For each $t_{j}$, denote the values of the discrete model by $\widetilde{y}_{j}=\left(u_{0}^{j}, u_{1}^{j}, u_{2}^{j}, \ldots u_{N-1}^{j}, u_{N}^{j}\right)^{\prime} \in \tilde{Y}$, where' means transpose and $\widetilde{Y}$ is a vector space of dimension $N+1$ with the norm

$$
\left\|\widetilde{y}_{n}\right\|_{\widetilde{Y}}^{2}=\sum_{i=0}^{N}\left(\widetilde{y}_{n}^{i}\right)^{2} .
$$

In the space $\widetilde{Y}$, we introduce operators $A$ and $\widetilde{A}$ :

$$
\begin{gathered}
A u_{i}^{j}=-a^{2} \frac{u_{i-1}^{j}-2 u_{i}^{j}+u_{i+1}^{j}}{h^{2}}, \quad 1 \leq i \leq N-1, \quad A u_{0}^{j}=0, \quad A u_{N}^{j}=0, \\
\widetilde{A}=\Delta^{2} A .
\end{gathered}
$$

Then, system (2.1) can be written in the form

$$
\frac{\widetilde{y}_{j+1}-2 \widetilde{y}_{j}+\widetilde{y}_{j-1}}{\Delta^{2}}+s A \widetilde{y}_{j+1}+s A \widetilde{y}_{j-1}+(1-2 s) A \widetilde{y}_{j}=F^{j}(v(\cdot)),
$$

where $F^{j}(v(\cdot))=\left(F_{0}^{j}\left(v_{0}(\cdot)\right), F_{1}^{j}\left(v_{1}(\cdot)\right), F_{2}^{j}\left(v_{2}(\cdot)\right), \ldots, F_{N-1}^{j}\left(v_{N-1}(\cdot)\right), F_{N}^{j}\left(v_{N}(\cdot)\right)\right)^{\prime}, v(\cdot)=I\left(\left\{\widetilde{y}_{k}\right\}_{j}\right) \in$ $Q^{N+1}[-\tau, \Delta]$. Denote by $Q^{N+1}[-\tau, \Delta]$ the space of vector functions with components from $Q[-\tau, \Delta]$.

Let us apply the identity

$$
\widetilde{y}_{j+1}=2 \widetilde{y}_{j}+\Delta^{2} \frac{\widetilde{y}_{j+1}-2 \widetilde{y}_{j}+\widetilde{y}_{j-1}}{\Delta^{2}}-\widetilde{y}_{j-1}
$$

and introduce the operators

$$
\begin{gathered}
R=\frac{1}{\Delta^{2}} E+s A, \\
\widetilde{R}=\Delta^{2} R=E+s \widetilde{A} .
\end{gathered}
$$


Note that the operators $\widetilde{A}(3.3)$ and $\widetilde{R}(3.6)$, in contrast to the operators $A(3.2)$ and $R(3.5)$, do not depend on either $h$ or $\Delta$; they only depend on $\sigma=a^{2} \Delta^{2} / h^{2}$.

Transform equation (3.4) to the form

$$
R\left(\widetilde{y}_{j+1}-2 \widetilde{y}_{j}+\widetilde{y}_{j-1}\right)+A \widetilde{y}_{j}=F^{j}(v(\cdot)) .
$$

Equation (3.7) is solvable with respect to $\widetilde{y}_{j+1}$ for any admissible weight $s$ (there exists $R^{-1}$ ); hence, we can write equation (3.7) in the explicit form

$$
\widetilde{y}_{j+1}=2 \widetilde{y}_{j}-\widetilde{y}_{j-1}-R^{-1} A \widetilde{y}_{j}+R^{-1}\left(F^{j}(v(\cdot))\right) \text {. }
$$

Introduce the vector $y_{j}=\left(y_{j}^{1}, y_{j}^{2}\right)^{\prime}=\left(\widetilde{y}_{j-1}, \widetilde{y}_{j}\right)^{\prime} \in Y$, where $Y=\tilde{Y} \oplus \tilde{Y}$ is a vector space of dimension $q=2(N+1)$. If the space $\widetilde{Y}$ is equipped with a norm, then the norm of the space $Y$ is defined as follows:

$$
\|y\|_{Y}^{2}=\left\|y^{1}\right\|_{\widetilde{Y}}^{2}+\left\|y^{2}\right\|_{\widetilde{Y}}^{2} .
$$

Relation (3.8) can be rewritten in the form $y_{j+1}^{1}=y_{j}^{2}, y_{j+1}^{2}=2 y_{j}^{2}-y_{j}^{1}-R^{-1} A y_{j}^{2}+R^{-1}\left(F^{j}(v(\cdot))\right)$. As a result, we obtain the difference scheme

$$
y_{j+1}=S y_{j}+\Delta \Phi\left(t_{j}, I\left(\left\{y_{k}\right\}_{j}\right), \Delta\right)
$$

where $S=\left(\begin{array}{cc}0 & 1 \\ ,-1 & 2-R^{-1} A\end{array}\right)$ and $\Phi\left(t_{j}, I\left(\left\{y_{k}\right\}_{j}\right), \Delta\right)=\left(\begin{array}{c}0 \\ R^{-1} F^{j}\left(I\left(\left\{y_{k}^{2}\right\}_{j}\right)\right) / \Delta\end{array}\right)$.

Define the function of exact values [4] for scheme (3.10) by the relations $z_{j}=\left(z_{j}^{1}, z_{j}^{2}\right)^{\prime}=$ $\left(\widetilde{z}_{j-1}, \widetilde{z}_{j}\right)^{\prime}$ and $\widetilde{z}_{j}=\left(u\left(x_{0}, t_{j}\right), u\left(x_{1}, t_{j}\right), \ldots, u\left(x_{N-1}, t_{j}\right), u\left(x_{N}, t_{j}\right)\right)^{\prime}$.

A function $\left\{t_{j}=t_{0}+j \Delta \in\left[t_{0}-\tau, T\right], j=-m, \ldots, 0\right\} \rightarrow Y: y\left(t_{j}\right)=y_{j}$ will be called starting values [4] of the model.

We say that starting values of the model have order $\Delta^{p_{1}}+h^{p_{2}}$ if there exists a constant $C$ independent of $z_{j}, y_{j}, \Delta, h$ such that $\left\|z_{j}-y_{j}\right\|_{Y} \leq C\left(\Delta^{p_{1}}+h^{p_{2}}\right), j=-m, \ldots, 0$.

For example, starting values of model (3.10) can be found as follows:

$$
y_{j}=z_{j}=\left(z_{j}^{1}, z_{j}^{2}\right)^{\prime}=\left(\widetilde{z}_{j-1}, \widetilde{z}_{j}\right)^{\prime}, \quad \widetilde{z}_{j}=\left(\varphi\left(x_{0}, t_{j}\right), \varphi\left(x_{1}, t_{j}\right), \ldots, \varphi\left(x_{N}, t_{j}\right)\right)^{\prime}, \quad j=-m, \ldots, 0 .
$$

Then, $p_{1}=\infty$ and $p_{2}=\infty$.

The error of approximation (the residual) with interpolation in the general difference scheme $[2-4]$ is calculated by the formula

$$
d_{n}=\left(z_{n+1}-S z_{n}\right) / \Delta-\Phi\left(t_{n}, I\left(\left\{z_{i}\right\}_{-m}^{n}\right), \Delta\right), \quad n=0, \ldots, M-1 .
$$

We say that a method has error order $\Delta^{p_{1}}+h^{p_{2}}$ for approximation with interpolation if there exists a constant $C$ independent of $d_{n}, \Delta, h$ such that $\left\|d_{n}\right\| \leq C\left(\Delta^{p_{1}}+h^{p_{2}}\right), n=1, \ldots, M$.

This definition of residual differs from the earlier introduced definition of residual without interpolation (2.2). However, the following statement is valid.

Theorem 2. Suppose that the residual in the sense of (2.2) has order $\Delta^{p_{1}}+h^{p_{2}}$, the functions $F_{i}^{j}$ are Lipschitz, the interpolation-extrapolation operator $I$ has error order $p_{0}$ on the exact solution, and $\sigma$ is fixed. Then, the residual with interpolation in the sense of (3.11) has the same error order with respect to $\Delta$ and $h$, and this order is $\Delta^{\min \left\{p_{0}, p_{1}, p_{2}\right\}+1}$. 
Proof. Consider the norms of the coordinates of residual (3.11):

$$
\begin{gathered}
\left\|d_{n}^{1}\right\|_{\widetilde{Y}}^{2}=\left\|\left(z_{n+1}^{1}-z_{n}^{2}\right) / \Delta\right\|^{2}=\left\|\frac{\widetilde{z}_{n}-\widetilde{z}_{n}}{\Delta}\right\|^{2}=0, \\
\left\|d_{n}^{2}\right\|_{\widetilde{Y}}^{2}=\left\|\left(z_{n+1}^{2}+z_{n}^{1}-2 z_{n}^{2}+R^{-1} A z_{n}^{2}\right) / \Delta-\frac{1}{\Delta} R^{-1}\left(F^{n}\left(I\left(\left\{z_{k}^{2}\right\}_{n}\right)\right)\right)\right\|^{2} \\
=\left\|\frac{\widetilde{z}_{n+1}+\widetilde{z}_{n-1}-2 \widetilde{z}_{n}}{\Delta}+\frac{1}{\Delta} R^{-1} A \widetilde{z}_{n}-\frac{1}{\Delta} R^{-1}\left(F^{n}\left(I\left(\left\{\widetilde{z}_{k}\right\}_{n}\right)\right)\right)\right\|^{2} \\
=\Delta^{2} \| \frac{\widetilde{z}_{n+1}-2 \widetilde{z}_{n}+\widetilde{z}_{n-1}}{\Delta^{2}}+\frac{1}{\Delta^{2}} R^{-1} A \widetilde{z}_{n}-\frac{1}{\Delta^{2}} R^{-1}\left(F^{n}\left(I\left(\left\{\widetilde{z}_{k}\right\}_{n}\right)\right) \|^{2} .\right.
\end{gathered}
$$

Hence, by the definition of the operators $\widetilde{R}(3.6)$ and $\widetilde{A}(3.3)$

$$
\begin{gathered}
\left\|d_{n}^{2}\right\|_{\widetilde{Y}}^{2}=\Delta^{2} \| \frac{\widetilde{z}_{n+1}-2 \widetilde{z}_{n}+\widetilde{z}_{n-1}}{\Delta^{2}}+\frac{1}{\Delta^{2}} \widetilde{R}^{-1} \widetilde{A} \widetilde{z}_{n}-\widetilde{R}^{-1}\left(F ^ { n } \left(I\left(\left\{\widetilde{z}_{k}\right\}_{n}\right) \|^{2}\right.\right. \\
=\Delta^{2}\left\|\widetilde{R}^{-1}\right\|^{2}\left\|\widetilde{R}\left(\frac{\widetilde{z}_{n+1}-2 \widetilde{z}_{n}+\widetilde{z}_{n-1}}{\Delta^{2}}\right)+\frac{1}{\Delta^{2}} \widetilde{A} \widetilde{z}_{n}-F^{n}\left(I\left(\left\{\widetilde{z}_{k}\right\}_{n}\right)\right)\right\|^{2}
\end{gathered}
$$

Then, by definition (3.1) of the norm in $\tilde{Y}$,

$$
\begin{gathered}
\left\|\widetilde{R}\left(\frac{\widetilde{z}_{n+1}-2 \widetilde{z}_{n}+\widetilde{z}_{n-1}}{\Delta^{2}}\right)+\frac{1}{\Delta^{2}} \widetilde{A} \widetilde{z}_{n}-F^{n}\left(I\left(\left\{\widetilde{z}_{k}\right\}_{n}\right)\right)\right\|_{\widetilde{Y}}^{2} \\
=\| \frac{\widetilde{z}_{n+1}-2 \widetilde{z}_{n}+\widetilde{z}_{n-1}}{\Delta^{2}}+s \widetilde{A}\left(\frac{\widetilde{z}_{n+1}-2 \widetilde{z}_{n}+\widetilde{z}_{n-1}}{\Delta^{2}}\right)+\frac{1}{\Delta^{2}} \widetilde{A} \widetilde{z}_{n}-F^{n}\left(I\left(\left\{\widetilde{z}_{k}\right\}_{n}\right) \|_{\widetilde{Y}}^{2}\right. \\
=\| \frac{\widetilde{z}_{n+1}-2 \widetilde{z}_{n}+\widetilde{z}_{n-1}}{\Delta^{2}}+\frac{1}{\Delta^{2}}\left(s \widetilde{A} \widetilde{z}_{n+1}+(1-2 s) \widetilde{A} \widetilde{z}_{n}+s \widetilde{A} \widetilde{z}_{n-1}\right)-F^{n}\left(I\left(\left\{\widetilde{z}_{k}\right\}_{n}\right) \|_{\widetilde{Y}}^{2}\right. \\
=\sum_{i=1}^{N-1} \mid \frac{u\left(x_{i}, t_{n+1}\right)-2 u\left(x_{i}, t_{n}\right)+u\left(x_{i}, t_{n-1}\right)}{\Delta^{2}}-s a^{2} \frac{u\left(x_{i+1}, t_{n+1}\right)-2 u\left(x_{i}, t_{n+1}\right)+u\left(x_{i-1}, t_{n+1}\right)}{h^{2}} \\
-s a^{2} \frac{u\left(x_{i+1}, t_{n-1}\right)-2 u\left(x_{i}, t_{n-1}\right)+u\left(x_{i-1}, t_{n-1}\right)}{h^{2}}-\left.F_{i}^{n}\left(I\left(\left\{u_{i}^{k}\right\}^{n}\right)\right)\right|^{2} .
\end{gathered}
$$

Let us estimate each term in the sum in (3.13) using the assumptions of the theorem:

$$
\begin{gathered}
\mid \frac{u\left(x_{i}, t_{n+1}\right)-2 u\left(x_{i}, t_{n}\right)+u\left(x_{i}, t_{n-1}\right)}{\Delta^{2}}-s a^{2} \frac{u\left(x_{i+1}, t_{n+1}\right)-2 u\left(x_{i}, t_{n+1}\right)+u\left(x_{i-1}, t_{n+1}\right)}{h^{2}} \\
-(1-2 s) a^{2} \frac{u\left(x_{i+1}, t_{n}\right)-2 u\left(x_{i}, t_{n}\right)+u\left(x_{i-1}, t_{n}\right)}{h^{2}} \\
-s a^{2} \frac{u\left(x_{i+1}, t_{n-1}\right)-2 u\left(x_{i}, t_{n-1}\right)+u\left(x_{i-1}, t_{n-1}\right)}{h^{2}}-F_{i}^{n}\left(I\left(\left\{u_{i}^{k}\right\}^{n}\right)\right) \pm F_{i}^{n}\left(u_{t_{n}}\left(x_{i}, \cdot\right)\right) \mid \\
\leq\left|\psi_{i}^{n}\right|+\left|F_{i}^{n}\left(u_{t_{n}}\left(x_{i}, \cdot\right)\right)-F_{i}^{n}\left(I\left(\left\{u_{i}^{k}\right\}^{n}\right)\right)\right|
\end{gathered}
$$

PROCEEDINGS OF THE STEKLOV INSTITUTE OF MATHEMATICS $\quad$ Vol. $281 \quad$ Suppl. 12013 


$$
\leq C_{1}\left(\Delta^{p_{1}}+h^{p_{2}}\right)+L_{F}\left\|u_{t_{n}}\left(x_{i}, \cdot\right)-I\left(\left\{u_{i}^{k}\right\}^{n}\right)\right\|_{Q} \leq C_{1}\left(\Delta^{p_{1}}+h^{p_{2}}\right)+L_{F} C_{2} \Delta^{p_{0}} .
$$

Thus, (3.12)-(3.14) yield

$$
\begin{gathered}
\left\|d_{n}\right\|_{Y}^{2} \leq \Delta^{2}\left\|\widetilde{R}^{-1}\right\|^{2} \sum_{i=1}^{N-1}\left(C_{1}\left(\Delta^{p_{1}}+h^{p_{2}}\right)+L_{F} C_{2} \Delta^{p_{0}}\right)^{2}=\Delta^{2}\left\|\widetilde{R}^{-1}\right\|^{2}(N-1)\left(C_{1}\left(\Delta^{p_{1}}+h^{p_{2}}\right)+L_{F} C_{2} \Delta^{p_{0}}\right)^{2} \\
\leq \Delta^{2}\left(C_{3} \Delta^{\min \left\{p_{1}, p_{0}\right\}}+C_{4} h^{p_{2}}\right)^{2}=\Delta^{2}\left(C_{3} \Delta^{\min \left\{p_{1}, p_{0}\right\}}+C_{4}(a \Delta / \sqrt{\sigma})^{p_{2}}\right)^{2}
\end{gathered}
$$

where $C_{3}=\left\|\widetilde{R}^{-1}\right\| \sqrt{N-1}\left(C_{1}+L_{F} C_{2}\right)$ and $C_{4}=\left\|\widetilde{R}^{-1}\right\| \sqrt{N-1} C_{1}$.

Therefore, $\left\|d_{n}\right\|_{Y} \leq C \Delta^{\min \left\{p_{0}, p_{1}, p_{2}\right\}+1}$, where $C=C_{3}+C_{4}(a / \sqrt{\sigma})^{p_{2}}$.

The theorem is proved.

To investigate the stability of the scheme, we apply the results of $[5,6]$. For this, we consider the homogeneous difference scheme corresponding to (3.7):

$$
R\left(\widetilde{y}_{j+1}-2 \widetilde{y}_{j}+\widetilde{y}_{j-1}\right)+A \widetilde{y}_{j}=0 .
$$

The operator A (3.2) is self-adjoint and positive [5] in the sense of the scalar product of the vectors $\widetilde{y}=\left(\widetilde{y}^{0}, \widetilde{y}^{1}, \ldots \widetilde{y}^{N}\right) \in \widetilde{Y}$ and $\widetilde{w}=\left(\widetilde{w}^{0}, \widetilde{w}^{1}, \ldots \widetilde{w}^{N}\right) \in \widetilde{Y}$ :

$$
(\widetilde{y}, \widetilde{w})=\sum_{i=0}^{N} \widetilde{y}^{i} \widetilde{w}^{i} h .
$$

Then, the operator $R(3.5)$ is also self-adjoint and positive.

It is proved in [6] that, if scheme (3.15) satisfies the condition

$$
R>A / 4,
$$

then, for any initial conditions, the solution of (3.15) satisfies the inequality

$$
\left\|\widetilde{y}_{n+1}\right\|_{*} \leq\left\|\widetilde{y}_{n}\right\|_{*}
$$

where

$$
\left\|\widetilde{y}_{n}\right\|_{*}=\frac{1}{4}\left(A\left(\widetilde{y}_{n}+\widetilde{y}_{n-1}\right), \widetilde{y}_{n}+\widetilde{y}_{n-1}\right)+\left(\left(R-\frac{1}{4} A\right)\left(\widetilde{y}_{n}+\widetilde{y}_{n-1}\right), \widetilde{y}_{n}+\widetilde{y}_{n-1}\right) .
$$

Now, consider the homogeneous scheme that corresponds to (3.10):

$$
y_{j+1}=S y_{j}
$$

It follows from inequality (3.17) that $\left\|y_{n+1}^{1}\right\|_{*} \leq\left\|y_{n}^{1}\right\|_{*}$ and $\left\|y_{n+1}^{2}\right\|_{*} \leq\left\|y_{n}^{2}\right\|_{*}$.

Then, by definition (3.9) of the norm in the space $Y$, we have $\left\|y_{n+1}\right\|_{Y}^{2}=\left\|y_{n+1}^{1}\right\|_{*}^{2}+\left\|y_{n+1}^{2}\right\|_{*}^{2} \leq$ $\left\|y_{n}^{1}\right\|_{*}^{2}+\left\|y_{n}^{2}\right\|_{*}^{2}=\left\|y_{n}\right\|_{Y}^{2}$.

Hence, $\left\|y_{n+1}\right\|_{Y} \leq\left\|y_{n}\right\|_{Y}$, which means that the estimate $\|S\| \leq 1$ holds for equation (3.18).

Thus, under condition (3.16), scheme (3.10) is stable in the sense of the definition given in [4].

Using (3.5), we can rewrite the operator condition of stability for the scheme under investigation in the form of the inequality (see [6])

$$
\frac{1}{\Delta^{2}} E+\left(s-\frac{1}{4}\right) A>0
$$


which means that, for any nonzero $\widetilde{y} \in \tilde{Y}$,

$$
\frac{1}{\Delta^{2}}(\widetilde{y}, \widetilde{y})+\left(s-\frac{1}{4}\right)(A \widetilde{y}, \widetilde{y})>0 .
$$

Since the largest eigenvalue of the operator $A(3.2)$ is estimated from above by the value $4 a^{2} / h^{2}[6]$, we have

$$
(\widetilde{y}, \widetilde{y})>\frac{h^{2}}{4 a^{2}}(A \widetilde{y}, \widetilde{y})
$$

Hence, inequality (3.19) will be valid if

$$
\frac{h^{2}}{4 a^{2} \Delta^{2}}+s-\frac{1}{4}>0
$$

Thus, scheme (3.10) is stable if

$$
s>\frac{1}{4}\left(1-\frac{1}{\sigma}\right)
$$

where $\sigma=a^{2} \Delta^{2} / h^{2}$.

For example, for the explicit scheme $(s=0)$, we obtain the well-known Courant condition $\sigma<1$; in the case of the implicit scheme $(s=1)$, the stability condition holds for any relation between the steps.

We have conducted the embedding into the general difference scheme with aftereffect [2-4]; now, using [4, Theorem 2], we obtain the following statement.

Theorem 3. Suppose that stability condition (3.20) holds, the residual in the sense of (2.2) has order $\Delta^{p_{1}}+h^{p_{2}}$, the functions $F_{i}^{j}$ are Lipschitz, the interpolation-extrapolation operator $I$ is Lipschitz continuous and has error order $p_{0}$ on the exact solution, the starting values have order $\Delta^{p_{3}}+h^{p_{4}}$, and $\sigma$ is fixed. Then, the method converges with order $\Delta^{\min \left\{\min \left\{p_{0}, p_{1}, p_{2}\right\}+1, p_{3}\right\}}+$ $h^{\min \left\{\min \left\{p_{0}, p_{1}, p_{2}\right\}+1, p_{4}\right\}}$.

\section{EXAMPLES OF NUMERICAL COMPUTATION}

Consider the equation with constant delay

$$
\frac{\partial^{2} u}{\partial t^{2}}=a^{2} \frac{\partial^{2} u}{\partial x^{2}}+a^{2} \pi^{2} e^{-t} \sin \pi x+e^{\tau-2 t} \sin ^{2} \pi x+u(x, t)(1-u(x, t-\tau)), 0 \leq t \leq 3,0 \leq x \leq 1,
$$

for $\tau=2$ and $a=1$ with initial conditions $u(x, t)=e^{-t} \sin \pi x,-\tau \leq t \leq 0,0 \leq x \leq 1$, and boundary conditions $u(0, t)=0, u(1, t)=0,0 \leq t \leq 3$.

The exact solution of the equation is $u(x, t)=e^{-t} \sin \pi x$.

Figure 1 shows the approximate solution of this equation by method $(2.1)$ with $F_{i}^{j}\left(v_{i, j}(\cdot)\right)=$ $f\left(t_{j}, x_{i}, u_{i}^{j}, v_{i, j}(\cdot)\right)$, piecewise linear interpolation [2] for $s=1$, and the numbers of partition points equal to 10 in $x$ and 60 in $t$. Figure 2 corresponds to $s=0$ with 21 partition points in $x$ and 60 partition points in $t$ (in this case, the stability condition does not hold). In Table 1, we present the norms of the differences between the matrices of exact and approximate solutions of equation (4.1) obtained for different values of the parameter $s$ and different steps. The norms of the differences were calculated by the formula

$$
\|U\|=\max _{0 \leq j \leq M} \sum_{i=0}^{N}\left|u\left(t_{j}, x_{i}\right)-u_{i}^{j}\right| .
$$




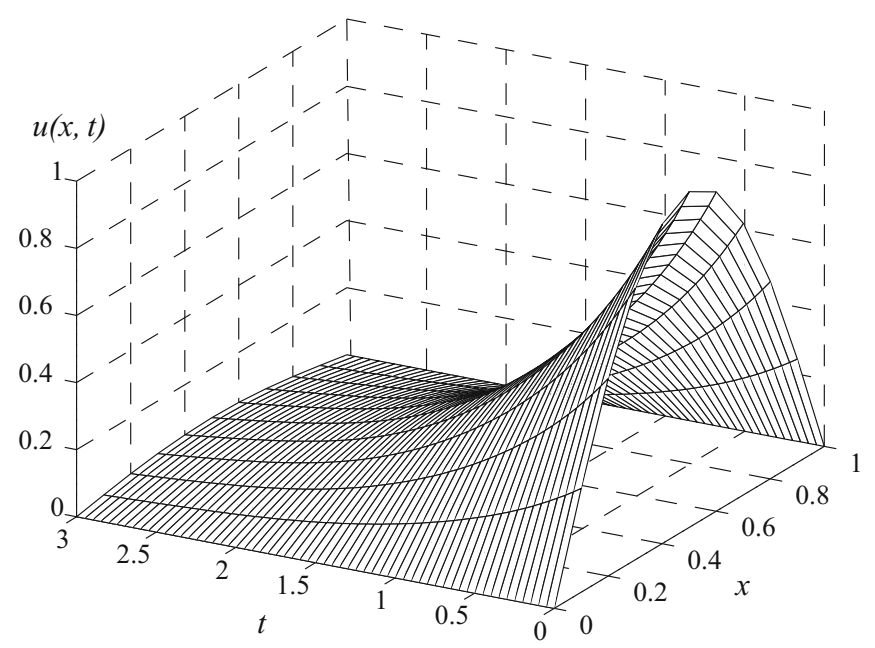

Fig. 1.

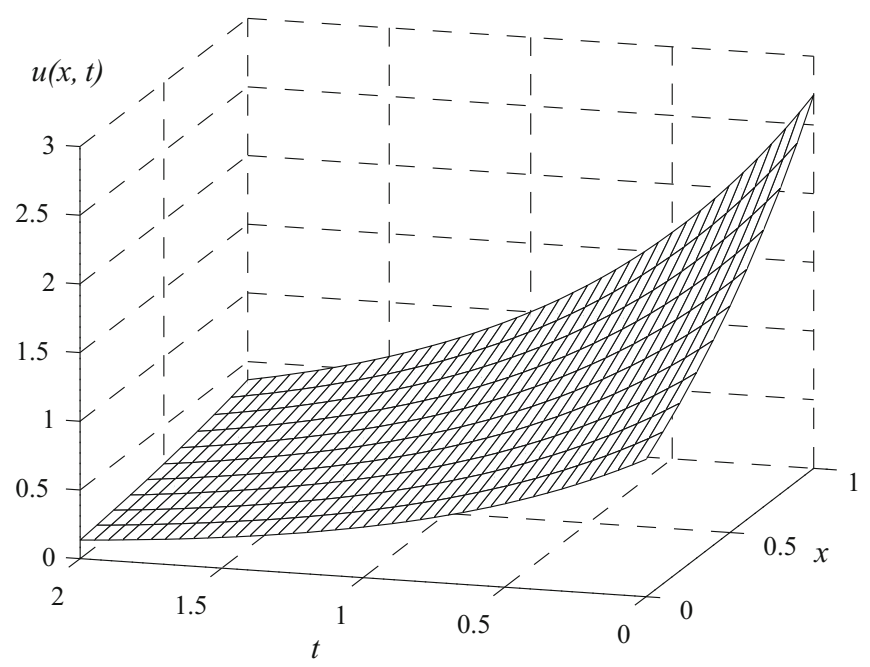

Fig. 2.

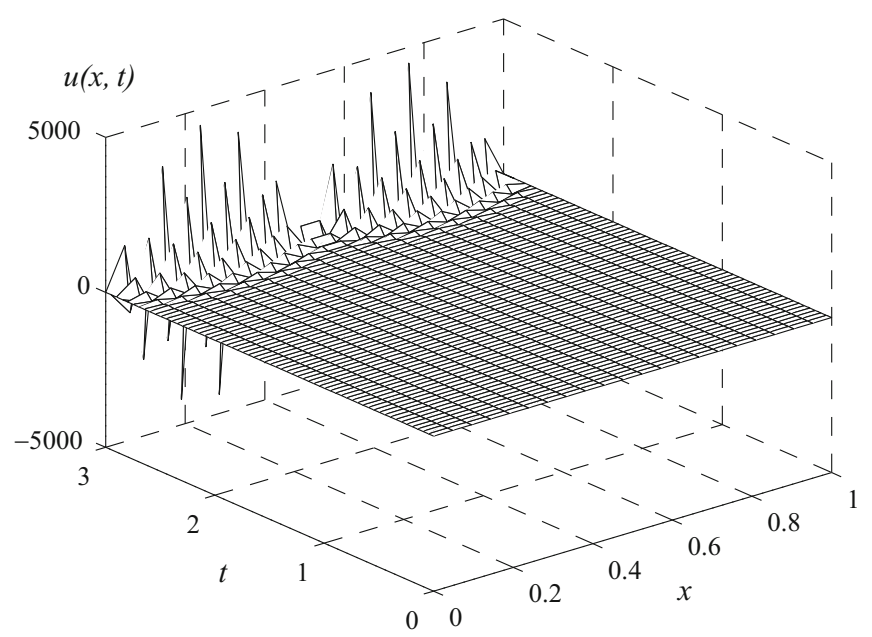

Fig. 3. 


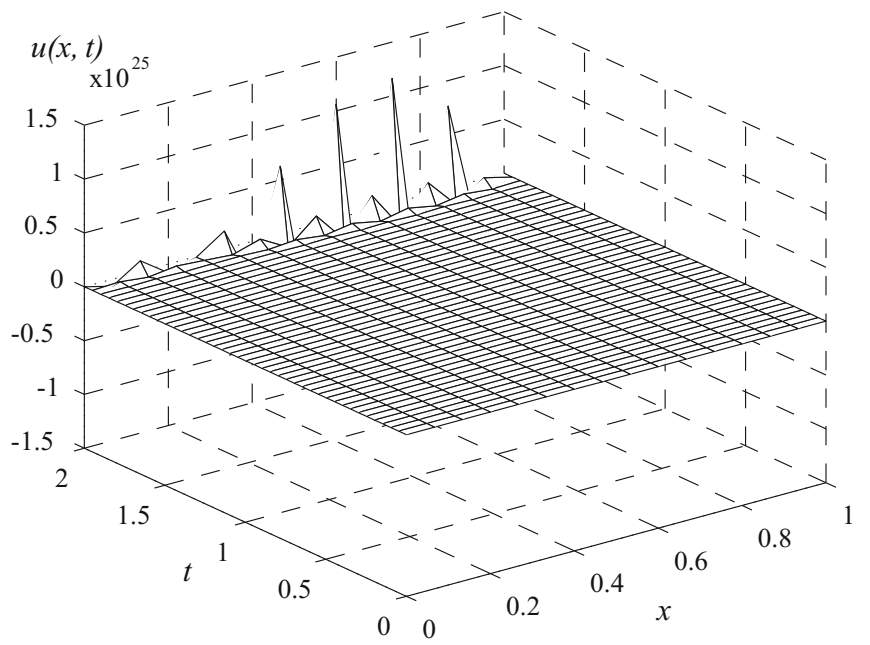

Fig. 4.

Consider the equation with varying delay

$$
\frac{\partial^{2} u}{\partial t^{2}}=a^{2} \frac{\partial^{2} u}{\partial x^{2}}+\left(1-a^{2}\right) \frac{u^{2}(x, t-(t+1) / 2)}{e^{x+1}}, \quad 0 \leq t \leq 2, \quad 0 \leq x \leq 1
$$

for $a=2$ with initial conditions $u(x, t)=e^{x-t},-1 \leq t \leq 0,0 \leq x \leq 1$, and boundary conditions $u(0, t)=e^{-t}, u(1, t)=e^{1-t}, 0 \leq t \leq 2$.

The exact solution of the equation is $u(x, t)=e^{x-t}$.

Figure 3 shows the approximate solution of this equation obtained by method (2.1) with $F_{i}^{j}\left(v_{i, j}(\cdot)\right)=f\left(t_{j}, x_{i}, u_{i}^{j}, v_{i, j}(\cdot)\right)$, piecewise linear interpolation [2] for $s=1$, and the numbers of partition points equal to 10 in $x$ and 40 in $t$. Figure 4 corresponds to $s=0$ with 15 partition points in $x$ and 40 partition points in $t$ (in this case, the stability condition does not hold). In Table 2, we present the norms of the differences between the matrices of exact and approximate solutions of equation (4.3) obtained for different values of the parameter $s$ and different steps. The norms of the differences were calculated by formula (4.2).

Table 1. Norms of differences between the exact and approximate solutions of equation (4.1)

\begin{tabular}{|c|c|c|c|c|c|}
\hline $\begin{array}{c}\text { Steps of the } \\
\text { grid method }\end{array}$ & $\begin{array}{c}N=10 \\
M=150\end{array}$ & $\begin{array}{c}N=10 \\
M=90\end{array}$ & $\begin{array}{c}N=10 \\
M=60\end{array}$ & $\begin{array}{c}N=15 \\
M=60\end{array}$ & $\begin{array}{c}N=21 \\
\text { s }\end{array}$ \\
\hline$s=1$ & 0.0502 & 0.0507 & 0.0527 & 0.0118 & 0.0095 \\
\hline$s=1 / 2$ & 0.0495 & 0.0488 & 0.0493 & 0.0248 & 0.0087 \\
\hline$s=0$ & 0.0488 & 0.0467 & 0.0443 & 0.0378 & 59952 \\
\hline
\end{tabular}

As the value for $s=0$ shows, the method has a large error in the case $N=21, M=60$.

Table 2. Norms of differences between the exact and approximate solutions of equation (4.3)

\begin{tabular}{|c|c|c|c|c|c|}
\hline $\begin{array}{c}\text { Steps of the } \\
\text { grid method }\end{array}$ & $\begin{array}{c}N=10 \\
M=200\end{array}$ & $\begin{array}{c}N=10 \\
M=100\end{array}$ & $\begin{array}{c}N=10 \\
M=60\end{array}$ & $\begin{array}{l}N=10 \\
M=40\end{array}$ & $\begin{array}{c}N=15 \\
M=40\end{array}$ \\
\hline$s=1$ & 0.4626 & 0.4625 & 0.4646 & 0.3265 & 0.0667 \\
\hline$s=1 / 2$ & 0.4629 & 0.4629 & 0.4660 & 0.3266 & 0.4670 \\
\hline$s=0$ & 0.4631 & 0.4635 & 0.4688 & 0.3276 & $7.888610 \times 10^{25}$ \\
\hline
\end{tabular}


As the value for $s=0$ shows, the method gives a large error in the case $N=15, M=40$.

These examples show that the errors of method (2.1) with condition (3.20) for certain values of the parameter $s$ depend strongly on the relation between the steps. If condition (3.20) does not hold, the error increases dramatically (see Figs. 2 and 4).

\section{ACKNOWLEDGMENTS}

This work was supported by the Russian Foundation for Basic Research (project no. 13-0100089).

\section{REFERENCES}

1. J. Wu, Theory and Applications of Partial Functional Differential Equations (Springer-Verlag, New York, 1996).

2. A. V. Kim and V. G. Pimenov, $i$-Smooth Analysis and Numerical Methods for Solving Functional Differential Equations (Regulyarn. Khaotichesk. Dinamika, Izhevsk, 2004) [in Russian].

3. V. G. Pimenov, Differential Equations 37 (1), 116 (2001).

4. V. G. Pimenov and A. B. Lozhnikov, Proc. Steklov Inst. Math. 275 (Suppl. 1), S137 (2011).

5. A. A. Samarskii, The Theory of Difference Schemes (Nauka, Moscow, 1977; Marcel Dekker, New York, 2001).

6. A. A. Samarskii and A. V. Gulin, Numerical Methods (Nauka, Moscow, 1989) [in Russian].

Translated by Ya. Salii 